\title{
Indicadores de Riesgo para la Caries Dental en Niños Preescolares de La Boquilla, Cartagena
}

\section{Risk indicators for dental caries in preschool children from La Boquilla, Cartagena}

Farith González-Martínez ${ }^{1}$, Ricardo Sánchez-Pedraza² y Luis Carmona- Arango ${ }^{3}$

1 Departamento de Investigación, Facultad de Odontología, Universidad de Cartagena. farithgm@hotmail.com

2 Facultad de Medicina, Universidad Nacional de Colombia. rsanchezpe@unal.edu.co

3 Departamento de Proyección Social, Facultad de Odontología, Universidad de Cartagena. leca1013@hotmail.com

Recibido 18 Diciembre 2008/Enviado para Modificación 7 Julio 2009/Aceptado 12 Julio 2009

\section{RESUMEN}

Objetivo Describir la ocurrencia de la caries en niños preescolares del corregimiento de La boquilla, Cartagena y relacionarlo con los factores reportados en la literatura como de riesgo.

Métodos La muestra fue de 238 niños entre 3 y 5 años. El promedio de edad fue 4.1 $(\mathrm{DE}=0.82)$. En cuanto al género, los niños fueron 116 y las niñas 122 . La selección individual fue realizada en forma probabilística proporcional al tamaño de la población de cada institución educativa. Para la evaluación de las variables explicatorias y el evento se utilizó un cuestionario y un instrumento clínico respectivamente y los datos fueron analizados a partir de las ocurrencias, realizando un análisis bivariado a través de los Odd Ratios y análisis multivariable por medio de regresión logística.

Resultados La prevalencia de caries fue de $60 \%$, siendo mayor para los niños de 5 años. Los factores asociados de mayor ocurrencia fueron los padres con escolaridad inferior a secundaria y la experiencia de caries. Al realizar los ajustes de los estimadores por la regresión logística, para la experiencia de caries se obtuvo un OR de 26,6, el consumo de dulces diariamente $\mathrm{OR}=3.37$ y la no exposición a fluorizaciones $\mathrm{OR}=2.17$.

Conclusiones La presencia de la caries en esta población se puede explicar por estos tres factores asociados; sin embargo, esta evidencia puede cambiar cuando estén involucrados otras circunstancias, momentos y lugares en niños con las mismas características.

Palabras Clave: Caries dental, riesgo, prevención primaria, preescolar (fuente: DeCS, BIREME).

\section{ABSTRACT}

Objective Describing the occurrence of dental caries in preschool children from the Boquilla neighbourhood in Cartagena and relating it to risk factors reported in the medical literature. 
Methods The sample consisted of 238 preschool children aged 3 to 5 . Average age was 4.1. Regarding gender, there were 116 boys and 122 girls. The children were individually selected in a probabilistic way, bearing in mind the size of the population from the chosen educational institution; a questionnaire and clinical instrument were used. The data was analysed according to outcome using Odd ratios for bivariate analysis and logistical regression for multivariable analysis.

Results Caries prevalence was $60 \%$, mainly occurring amongst 5 year old children. The major risk factors observed were parents having not progressed to more than secondary level studies and a background of caries. A 26.6 OR was obtained at the moment of adjusting the logistical regression estimators for having a background of caries, 3.37 OR for daily candy consumption and 2.17 OR for non-exposure to fluorides.

Conclusions The presence of caries in this population could be explained by means of the three associated risk factors mentioned above. This evidence may become changed when other circumstances, moments and places are involved in children having the same characteristics.

Key Words: Dental caries, risk, primary prevention, preschool child (source: $\mathrm{MeSH}$, NLM).

a caries dental se considera un problema de salud pública, ya que en el último estudio nacional de salud bucal en 1998 utilizando índices de caries tradicionales ceo/COP, se evidenció que el 65,8\% de los colombianos presentaron una o más lesiones. Al analizar los niños de cinco años, la prevalencia fue del $54.8 \%$. En la costa atlántica incluyendo a Cartagena, los datos fueron similares, reportándose una prevalencia del $55,6 \%$. Si bien estos datos muestran una importante disminución de la enfermedad entre 1978 y 1998, todavía no hay registros nacionales con seguimientos longitudinales amplios en edades menores de 5 años que lleven a complementar esta información. Por otro lado, no hay reportes de factores asociados que se aproximen al entendimiento de un modelo de riesgo $(1,2)$.

En este sentido, la valoración del riesgo para caries dental no puede asumirse mediante un modelo universal. Es importante resaltar que en la ciudad de Cartagena existen poblaciones con diversidad de exposiciones a los factores que reporta la literatura como indicadores de riesgo y esto podría estar influyendo en las dificultades que tienen los profesionales para poder mantenerlos sanos. Es por esto que se generó esta propuesta, ya que a pesar de que existe suficiente evidencia sobre el origen de la caries, estos estudios han sido realizados en otras poblaciones, las cuales tienen costumbres y estilos de vida diferentes que pueden representar discrepancias al momento de utilizarlos para valorar el riesgo colectivo e individual. 
Algunos estudios realizados en la población boquillera han aportado ciertos elementos de juicio para poder evidenciar una grande problemática, sin embargo, todo el interés se ha generado a partir de los seis años, dejando a un lado los niños en edad preescolar, los cuales presentan dentición temporal y tienen exposiciones diferentes a los factores de riesgo para Caries, lo que hace interesante construir un nuevo conocimiento enmarcado en un modelo de riesgo, que permita intervenir con éxito a temprana edad (3-5).

Desde esta perspectiva, el objetivo de este estudio fue describir la ocurrencia de la caries en niños preescolares del corregimiento de La boquilla - Cartagena de Indias y relacionarlo con los factores reportados en la literatura como de riesgo.

\section{MÉTODOS}

Para este diseño de corte transversal se efectuó un muestreo aleatorio simple, estratificado por institución preescolar, considerando asignación proporcional por el tamaño del estrato (hogares infantiles $30 \%$, instituciones educativas del distrito $51 \%$ y escuelas de congregaciones religiosas $19 \%$ ). Para el cálculo de tamaño de muestra se asumió un nivel de confianza del $95 \%$, una frecuencia esperada de la enfermedad en el grupo no expuesto del $54 \%$ y un OR estimado de 1,7 (el más pequeño obtenido de la literatura a partir de la variable placa bacteriana). Para conformar el marco muestral se tuvieron en cuenta a los niños residentes en el corregimiento, matriculados en alguna institución preescolar, sin enfermedades sistémicas ni alteraciones motrices. Así se obtuvo una muestra probabilística de 238 niños del corregimiento de La boquilla-Cartagena. La evaluación se realizó previa aceptación de los padres del menor, a través la firma de un consentimiento informado. Se utilizó un cuestionario para medir las variables explicatorias y un instrumento clínico para la valoración del evento caries y placa bacteriana.

Cuestionario: Para la información de los factores asociados a Caries se utilizó una encuesta estructurada dirigida a los padres, valorándose dieta cariogénica, responsabilidad del cepillado delegada en el niño, frecuencia de cepillado de 1 vez/día, no exposición a fluoruros, hijos de padres separados/madre soltera, escolaridad de los padres inferior a secundaria, asistencia a consulta odontológica menos de una vez/año y mala conducta escolar. Este cuestionario fue evaluado por tres expertos con experiencia en la construcción de éstos instrumentos para obtener la validez de apariencia. Posteriormente en una prueba piloto se verificó la validez de concepto, aplicando el cuestionario a 10 padres de una población con características similares, para determinar si las diferentes puntuaciones arrojadas por la prueba podían soportarse por la teoría y si éstas medían lo que 
realmente se quería medir. Además, para la valoración de la dieta, se utilizaron los criterios diagnósticos del Instituto Colombiano de Bienestar Familiar ICBF aplicados en la última encuesta nacional de nutrición en Colombia ENSIN 2005 (6). Para la recolección de la información de los factores asociados a caries, se convocó al padre del niño seleccionado que permanecía con él la mayor parte del tiempo. Previamente se diligenció un formato de consentimiento informado, conforme lo estipulado en la resolución 8430 de 1993 Ministerio de Salud (7). Este proyecto fue avalado por el comité de ética de la universidad de Cartagena.

Instrumento clínico: Para obtener la información de la variable considerada evento (caries) y los factores asociados (placa bacteriana y experiencia de caries - coe-d) se utilizó el Formato Guías de práctica clínica en salud oral, Secretaria Distrital de Salud, Bogotá- ACFO (8). Además, se utilizaron los criterios diagnósticos de caries (ICDAS II) (9). Para estandarizar este instrumento se realizó una prueba piloto con el fin de evaluar las diferencias intra e interexaminador. La concordancia entre las observaciones se realizó a través de un análisis Kappa Cohen, asumiendo un grado de acuerdo aceptable de 0,80 y 0,90 respectivamente con respecto a un patrón de oro. Los resultados de la prueba permitieron controlar el error de medición de los diagnósticos realizados por los dos examinadores de mejor puntaje. De la misma forma, este procedimiento se realizó para medir placa bacteriana, utilizando el Índice de Placa Comunitario IPC de Corchuelo 1996 (10).

Examen Clínico: La valoración clínica se inició con la medición de la higiene oral, mediante la aplicación de sustancia reveladora ditono a nivel de todas las superficies dentales; estas fueron manchadas con el fin de que la tinción quedará uniforme. Luego se clasificó el índice a nivel dicotómico: placa deficiente y regular $\geq 15 \%$ y placa buena $<15 \%$. Posteriormente se realizó el diagnóstico de caries. Previa profilaxis se observaron los dientes y superficies cariadas, permitiendo evaluar los cambios de color y las cavidades, utilizando los siguientes códigos: $0=$ superficie sana, $1=$ Primer cambio visual en esmalte (visto solo después de un secado prolongado con aire o circunscrito a los límites de una fosa o fisura), 2=Cambio visual marcado en esmalte, 3=Ruptura localizada en esmalte (Sin signos visuales clínicos de compromiso dentinal), 4=Sombra Oscura subyacente que se proyecta desde la dentina, $5=$ Cavidad marcada con exposición de la dentina, $6=$ Cavidad marcada amplia con exposición de la dentina.

Para evaluar las obturaciones se utilizaron los criterios de adaptación compatible con salud. Por último se evaluaron los dientes con extracción indicada, siendo incluidos solo aquellos que se habían perdido por caries. 
Análisis Estadístico: Se estimó la frecuencia de caries y de factores asociados a través de prevalencias. Los estimadores fueron calculados teniendo en cuenta el diseño muestral, utilizando la rutina "svy" del programa estadístico STATA9®. La fuerza de asociación fue calculada por medio de razones de disparidad (OR), con intervalos de confianza del $95 \%$, efectuando los correspondientes ajustes derivados del diseño muestral. Por último, se realizó un análisis multivariable mediante regresión logística, teniendo en cuenta estimadores crudos y ajustados por regresión (11).

\section{RESULTADOS}

El promedio de edad fue 4,1 (DE=0,82), de los cuales 73 correspondían a niños de tres años, 77 de cuatro y 88 de cinco años. En cuanto al género, los niños fueron 116 y las niñas 122. Se encontró una prevalencia de caries de $60 \%$ (IC $95 \%$ : 53-67), siendo $72 \%$ para los hogares infantiles (IC 95\%: 66-77) y $56 \%$ para las instituciones de congregaciones religiosas (IC $95 \%$ : 49-62). El promedio de coe-s fue de 5,3 (IC $95 \%: 4,6-5,9 ; \mathrm{DE}=5,3)$. La mayor prevalencia de caries fue para los niños de cinco años con $74 \%$ (IC 95\%: 68-79; p=0,02) y un ceos de 5,6 (IC 95\%: 5,1-6,1; DE= 5 ,6). En cuanto al género, la mayor frecuencia fue en varones con $66 \%$ (IC $95 \%$ : 59-72; $\mathrm{p}=0,19$ ) (Tabla 1).

Tabla 1. Ocurrencia y promedio de caries de acuerdo con variables

\begin{tabular}{|c|c|c|c|c|c|c|}
\hline & $N$ & $\begin{array}{c}\text { Prevalencia* } \\
\%\end{array}$ & $\begin{array}{c}\text { IC } 95 \% \\
\text { prevalencia }\end{array}$ & $\begin{array}{l}\text { Media } \\
\text { coe-s }\end{array}$ & $\begin{array}{c}\mathrm{DE} \\
\text { coe-s }\end{array}$ & $\begin{array}{c}\text { IC 95\% } \\
\text { coe-s }\end{array}$ \\
\hline \multicolumn{7}{|l|}{ Género } \\
\hline Masculino & 77 & 66 & $59-72$ & 5,7 & 5,3 & $5,3-6,1$ \\
\hline Femenino & 71 & 58 & $51-64$ & 4,9 & 5,4 & $4,5-5,3$ \\
\hline \multicolumn{7}{|l|}{ Edad } \\
\hline 3 & 44 & 60 & $56-66$ & 5,3 & 5,2 & $4,7-5,8$ \\
\hline 4 & 39 & 50 & $43-57$ & 4,9 & 5,2 & $4,4-5,4$ \\
\hline 5 & 65 & $74^{* *}$ & $68-79$ & 5,6 & 5,7 & $5,1-6,1$ \\
\hline \multicolumn{7}{|l|}{$\begin{array}{l}\text { Tipo de } \\
\text { Institución }\end{array}$} \\
\hline Hogares & 33 & $72 * *$ & $66-77$ & 5,5 & 5,0 & $4,9-5,9$ \\
\hline Inst. Distrital & 76 & 62 & $56-68$ & 5,5 & 6,0 & $5,0-10,0$ \\
\hline Inst. Religiosa & 39 & 56 & $49-62$ & 5,2 & 4,3 & $4,6-9,8$ \\
\hline Total & 148 & 60 & $53-67$ & 5,3 & 5,4 & $4,6-5,9$ \\
\hline
\end{tabular}

Con respecto los factores asociados a caries, los de mayor prevalencia fueron la escolaridad de los padres inferior a secundaria (69 \%; IC $95 \%$ : 63\%-75\%), experiencia de caries (67\%; IC $95 \%: 61 \%-73 \%$ ), índice de placa deficiente (64\% ; IC $95 \%$ : $58 \%-61 \%$ ), la no exposición a fluorizaciones (58\%; IC $95 \%$ : 
$51 \%-65 \%$ ), el consumo de dulces diariamente (54\%; IC $95 \%$ : $47 \%$ - $61 \%$ ) y la dieta cariogénica (52\%; IC $95 \%$ : $45 \%-69 \%$ ) (Tabla 2).

Tabla 2. Ocurrencia de indicadores asociados a caries

\begin{tabular}{lccc}
\hline \multicolumn{1}{c}{ Factores asociados } & Número & Prevalencia (\%) & IC 95\% \\
\hline Experiencia de caries & 162 & 67 & $61-73$ \\
Placa bacteriana > 15\% & 143 & 64 & $58-71$ \\
Dieta cariogénica & 133 & 52 & $45-59$ \\
Consumo diario de dulces & 114 & 54 & $47-61$ \\
El niño es responsable del cepillado & 114 & 50 & $43-57$ \\
Frecuencia de cepillado una vez al día & 48 & 23 & $17-29$ \\
Consulta odontológica > 1 vez-año & 114 & 50 & $43-57$ \\
No exposición a flúor & 92 & 58 & $51-65$ \\
Escolaridad de padres < secundaria & 154 & 69 & $63-75$ \\
Sin información padres higiene oral & 109 & 50 & $43-57$ \\
Padres separados/madre soltera & 78 & 31 & $25-37$ \\
Niños están sin padres durante el día & 18 & 8 & $4-11$ \\
Ninguna actividad recreativa & 32 & 17 & $11-23$ \\
Mala conducta escolar & 46 & 22 & $16-28$ \\
\hline
\end{tabular}

En el análisis bivariado se encontró asociación estadísticamente significativa entre la ocurrencia de caries y la experiencia de caries $(\mathrm{OR}=25,4$; IC $95 \%$ : 11,854,6; $\mathrm{p}<0.000001$ ), el consumo de dulces diariamente (OR=3,1; IC $95 \%: 1,7-5,3$; $\mathrm{p}<0.0001)$, los niños de padres separados (OR=2,3; IC 95\%: 1,2-4,3; $\mathrm{p}=0.01)$, y la no exposición a fluorizaciones $(\mathrm{OR}=2,0 ;$ IC $95 \%: 1,2-3,5 ; \mathrm{p}=0.01)$ (Tabla 3).

Tabla 3. Asociación entre caries e indicadores de riesgo

\begin{tabular}{|c|c|c|c|c|c|c|}
\hline \multirow[t]{2}{*}{ Factores asociados } & \multicolumn{2}{|c|}{ Caries } & \multicolumn{2}{|c|}{ Bivariado } & \multicolumn{2}{|c|}{ Multivariado } \\
\hline & $\mathrm{Si}$ & No & $\mathrm{OR}^{\#}$ & IC $95 \%$ & $\mathrm{OR}^{\# \#}$ & IC $95 \%$ \\
\hline Experiencia de caries & 135 & 27 & $25,4^{\star}$ & $11,8-54,6$ & $26,6^{*}$ & $11,9-59,5$ \\
\hline Placa bacteriana > $15 \%$ & 97 & 46 & 1,7 & $0,9-3,1$ & & \\
\hline Dieta cariogénica & 81 & 52 & 0,8 & $0,5-1,4$ & & \\
\hline Consumo diario de dulces & 91 & 39 & $3,1^{*}$ & $1,7-5,3$ & $3,4 \square$ & $1,6-7,1$ \\
\hline El niño es responsable del cepillado & 31 & 17 & 1,6 & $0,9-2,7$ & & \\
\hline Frecuencia de cepillado una vez/día & 31 & 17 & 1,1 & $0,6-2,3$ & & \\
\hline Consulta odontológica $>1$ vez/año & 78 & 36 & 1,5 & $0,9-2,6$ & & \\
\hline No exposición a flúor & 92 & 41 & $2,0^{\star \star}$ & $1,2-3,5$ & $2,2 \mathrm{an}$ & $1,1-4,5$ \\
\hline Escolaridad de padres $<$ secundaria & 96 & 58 & 0,9 & $0,5-1,7$ & & \\
\hline Sin información padres higiene oral & 66 & 43 & 0,7 & $0,4-1,2$ & & \\
\hline Padres separados/madre soltera & 58 & 20 & $2,3^{\star *}$ & $1,2-4,3$ & 2,1 & $0,9-4,8$ \\
\hline Niños sin padres durante el día & 12 & 6 & 1,2 & $0,4-3,9$ & & \\
\hline Ninguna actividad recreativa & 17 & 15 & 0,6 & $0,3-1,4$ & & \\
\hline Mala conducta escolar & 32 & 14 & 2,0 & $0,9-4,2$ & & \\
\hline
\end{tabular}

En cuanto al análisis multivariable a través de la regresión logística, el mejor modelo mostró asociaciones estadísticamente significativas que explican la aparición de la caries $(\mathrm{p}<0.00001)$ entre tres factores: la experiencia de caries $(\mathrm{OR}=26,6 ; \mathrm{p}<0.00001)$, el consumo de dulces diariamente $(\mathrm{OR}=3,4 ; \mathrm{p}<0.001)$ y 
la no exposición a fluorizaciones $(\mathrm{OR}=2,2 ; \mathrm{P}=0.03)$ (Tabla 3). $\mathrm{Al}$ realizar la corrección de los estimadores para aproximarlos al riesgo relativo, se obtuvo para la experiencia de caries un OR=4,9 (IC $95 \%: 2,8-7,2$ ), para el consumo de dulces diariamente un $\mathrm{OR}=1,5$ (IC $95 \%: 1,1-1,6$ ) y para la no exposición a fluorizaciones un $\mathrm{OR}=1,3$ (IC $95 \%: 1,1-1,6$ )

\section{DISCUSIÓN}

El impacto de poner a prueba diferentes factores de riesgo para las asociaciones con la caries dental está fundamentado en la obtención de un modelo de riesgo de la enfermedad para futuras intervenciones, siendo los resultados útiles para tomar decisiones de salud pública al momento de plantear estrategias preventivas en poblaciones vulnerables. Este modelo sería exitoso en la medida en que sea factible su aplicación desde la operatividad de los programas preventivos que se impulsan en esta región.

La ocurrencia de caries observada en esta población se considera alta, debido a que todavía no se cumple con las metas en dentición temporal trazadas por la OMS para el año 2000. Además, al comparar los resultados de prevalencia de caries a nivel local, estos son similares a los reportados por González (12) en niños de la misma edad del ICBF de Cartagena, lo que puede coincidir con sus hábitos de higiene oral y la influencia sociocultural de los individuos de Cartagena. Por otro lado en niños de las ciudades capitales como Bogotá (13) y Medellín, (14) los resultados fueron mayores a los observados en el presente estudio, diferencias que se pueden explicar debido a que en estas dos últimas poblaciones se utilizaron por primera vez en el país los nuevos criterios diagnósticos de caries para análisis epidemiológico y el tiempo transcurrido después de ocho años con la instauración de innumerables acciones preventivas puede haber influido en la variación de la prevalencia de la enfermedad a nivel local. En este sentido, es clara la susceptibilidad de los niños menores de cinco años, debido a que por su corta edad no son autónomos en la utilización de herramientas preventivas. Por otra parte, la mayor prevalencia de caries para la edad de cinco años ha sido reportada en diferentes estudios debido a que para esta edad el niño incrementa las exposiciones a factores como la ingesta de dulces, inicia la etapa de erupción de los primeros dientes permanentes, ocasionándole incomodidades durante el cepillado dental y además los padres terminan delegándoles la responsabilidad de este como una medida para generar autonomía e interés en su higiene oral (15).

En cuanto a las asociaciones encontradas en el presente estudio, se han obtenido resultados similares en varias poblaciones a nivel mundial. En primera 
instancia en Colombia la experiencia de caries ha sido el indicador de mayor fuerza estadística, encontrándose OR de 4,8 en niños preescolares de Boyacá (16) y de 18 en niños preescolares de la ciudad de Cartagena (17). A nivel mundial Powell (18) a través de una revisión sistemática de varios modelos predictores de caries encontró que la experiencia pasada de caries en dentición temporal es uno de los mejores indicadores del riesgo, especialmente las lesiones de los incisivos primarios. En este aspecto se sabe que una lesión cavitacional ofrece un ambiente favorable para que se retenga la placa bacteriana.

Con respecto al consumo de dulces diariamente, los estimadores de asociación fueron similares a otras poblaciones. Según Vanobberggen (19) los niños que ingieren "bocadillos" más de dos veces por día entre las comidas tienen 1,3 veces más probabilidad de desarrollar caries. Por otro lado. Declerck (20) reportó que los niños que consumen bebidas azucaradas entre las comidas e ingesta de meriendas en las noches tienen 2,6 veces más probabilidad de presentar caries. Desde esta perspectiva, la ingesta de azúcares entre comidas aumenta el pH del medio oral ocasionando un disturbio adicional al de la presencia natural de la placa bacteriana, de esta forma, se disminuyen las posibilidades de que se remineralicen las lesiones con los iones minerales aportados por la saliva (21). Esto es ratificado en un estudio realizado en niños Finlandeses por Karjalainen (22) en donde la proporción de niños con una combinación de ingesta de dulces de más de una vez a la semana y placa visible a los 3 años, incrementaron la probabilidad de desarrollar caries a los 6 años $\mathrm{OR}=1,7$. En contraposición con estos resultados, Anderson (23) en un estudio sobre detección de caries interproximal en niños preescolares de Suecia encontró que la ingesta de productos azucarados entre las comidas es un predictor con moderada precisión de las lesiones de caries. Por último Pienihakkinen (24)en niños de Finlandia concluyó que la combinación de lesiones de caries incipientes y el consumo de caramelos en preescolares tiene suficiente valor predictivo para las implicaciones clínicas de la enfermedad.

El uso frecuente de complementos locales de flúor diferentes al de las cremas dentales también fue un factor con fuerza estadística en el presente estudio; esto se debe probablemente a que en poblaciones con múltiples exposiciones, el flúor tópico durante la fluorización al menos dos veces al año se convierte en una herramienta que contribuye a remineralizar las nuevas lesiones. Desde esta perspectiva, Perinetti (25) encontró en niños entre 2 y 5 años de 13 provincias de Italia, que el uso regular de suplementos de flúor resulta ser protector $(\mathrm{OR}=0,58)$ para desarrollar caries dental. 
En cuanto al riesgo que representan los hogares con padres separados o madre soltera, estos resultados son similares a los obtenidos por Schwartz (26) el cual refiere que el rompimiento del núcleo familiar es un componente de mayor estrés psicosocial y este puede deberse a muerte de alguno de los padres, separación o divorcio de la pareja, afectándose el comportamiento de los niños, viéndose reflejado en su salud. En este aspecto, según Febres (27) y Finlayson (28) es característico encontrar en ausencia de autoridad de los padres mucha manipulación y control, relaciones viciadas, sobreprotección y descuido. Además, la mayoría de los casos los niños que viven con un único padre conviven en circunstancias económicas inestables, disminuyéndose la posibilidad de tener acceso a servicios de salud e invertir en herramientas preventivas.

En lo que se refiere a la obtención de un modelo de riesgo, los factores asociados de mayor coincidencia con la literatura fueron el consumo de golosinas y bebidas azucaradas y la presencia de placa visible, debido a que los azucares juegan un papel trascendental en la velocidad de progresión de la desmineralización, acentuándose cuando hay presencia de placa patológica sin disturbio durante los primeros ocho días $(29,30)$.

Al realizar las interacciones entre placa y las tres variables de mayor significancia en el presente estudio, se evidencia un aumento en la asociación, siendo la dupla placa - consumo de dulces la de mayor fuerza (OR=12,8; IC $95 \%$ : 6,02-26,9) Este aspecto hoy en día no es discutible, ya que la presencia de placa bacteriana sobre la superficie dental se considera el factor necesario para que se produzca la desmineralización. Además esto es reafirmado en un estudio realizado en niños preescolares en el 2002 por Dos santos (31) en el que al relacionar estas dos variables concluyen que la experiencia de azúcar en la dieta y los cambios bioquímicos y microbiológicos en la composición de la placa dental podrían explicar en parte las diferentes tipos de caries observadas en dentición temporal.

A la luz de las limitaciones de este diseño, se puede concluir que la ocurrencia de la caries en esta población está influida por múltiples variables, las cuales conjugadas en diferentes circunstancias, momentos y lugares pueden cambiar su interacción, dándole una lectura diferente al proceso etiológico de la enfermedad oral. Esto contribuye en forma ostensible a que no se hayan podido controlar los índices de caries en edades tempranas, por la poca evidencia que existe en la región de investigaciones que generen la construcción de estos modelos de riesgo * 
Agradecimientos. A la Facultad de Odontología de la Universidad de Cartagena por haber contribuido con la estructuración del trabajo de campo dentro de las acciones comunitarias proyectadas por el Departamento de Proyección Social.

\section{REFERENCIAS}

1. Ministerio de Salud - República de Colombia, Centro Nacional de Consultoría CNS: III- ENSAB III; Tomo VII. Bogotá: Lito Servicios ALER; 1999. p. 84-88.

2. Moncada H, Erazo B. Estudio Nacional de salud bucal. 1977-1980. INS. Ascofacme. Bogotá; 1983. p. 65-66.

3. González FD, Alfaro LM, Nieto C, Carmona LE. Evaluación de las condiciones de salud oral y los conocimientos en niños escolares entre 7 y 14 años de la población de La boquilla, Cartagena de Indias 2005. Revista Científica Facultad de Odontología Universidad El Bosque 2006; 12 (1): 25-34.

4. González FD, Alfaro LM, Nieto C. Relación de placa bacteriana y caries en niños escolarizados entre 5 y 14 años de la población de La boquilla, Cartagena. Rev. DUAZARY Universidad del Magdalena 2007; 4 (2): 119-126.

5. González FD, Oyola E, Cadrazco F, Herrera Y. Representaciones sociales sobre los escenarios de prácticas en salud oral en familiar del corregimiento de La boquilla, Cartagena 2005. Rev. CES Odontología 2007; 12 (1): 9-19.

6. Samper B, Manjarrez L, Álvarez M, Gómez L, Forero Y, Correa J, Estrada A, De Ossa G. Encuesta Nacional de la situación nutricional en Colombia ENSIN 2005. Instituto Colombiano de Bienestar Familiar. República de Colombia; 2005. p 65-66.

7. Normas Científicas, Técnicas y Administrativas para la Investigación en Seres Humanos. Resolución 008430 Ministerio de Salud. República de Colombia; 1993. p. 53-54.

8. Martignón S, González M, Mccornikc V, Ruiz JA, Jácome S, Guarnizo C. Guías de Diagnóstico, Prevención y Tratamiento de la Caries dental. Asociación Colombiana de Facultades de Odontología - ACFO., Secretaria Distrital de Salud Bogotá. Editor: Secretaria Distrital de Salud Bogotá, Bogotá; 2007. p. 77- 86.

9. Ismail Al, Sohn W, Tellez M, Amaya A, Sen A, Hasson H, Pitts NB. The International Caries Detection and Assessment System (ICDAS): and integrated system for measuring dental Caries. Community Dent Oral Epidemiol 2006; 34: 1-9.

10. Corchuelo J, Trillos Z. Sensibilidad de un nuevo indicador de placa dental de uso comunitario para el monitoreo de programas dirigidos a grupos del Plan de Atención Básica y del POS. Memorias ACFO; 1996; 102-109.

11. Zhang J, Kai F. What's the Relative Risk? A Method of Correcting the Odds Ratio in Cohort Studies of Common Outcomes. JAMA 1998; 280 (19): 1690-1691.

12. González FD, Luna LM, Martínez N, Solana M. Correlación entre los factores de riesgo biológicos y sociales con la presencia de caries dental en niños entre 5 y 6 años de cinco hogares infantiles del ICBF de la ciudad de Cartagena Rev. Federación Odontológica Colombiana 2007; 69 (219): 7-20.

13. González MC, Ruiz JA, Martignon E. Comparison of the def Index with Nyvad Caries Diagnostic Criteria in 3 and 45 years old Colombia Children. Pediatric Dentistry 2003; 25 (2): 132-136.

14. Saldarriaga A, Franco A, González S, Escobar A, Cordero N, Dávila H. Evaluación del subregistro de caries dental en dentición decidua. Rev. CES Odontología 2003; 16 (2): 43-47.

15. Tsai A, Chen Ch, Li L, Hsiang Ch, Hsu K. Risk Indicators for early childhood caries in Taiwan. Community Oral Dent Epidemiol 2006; 34: 437-445.

16. Rey M, Salas E, Martignón S. Caries dental y factores de riesgo asociados en la población escolar de Moniquirá, Boyacá. Rev. Científica Facultad de Odontología Universidad El Bosque 2003; 9 (2): 28-34. 
17. González FD, Luna LM, Martínez N, Solana M, Relación entre caries y factores de riesgo en niños preescolares del Bienestar Familiar Cartagena. Rev. Academia Colombiana de Odontología Pediátrica 2007; 5 (1): 60.

18. Powel V. Caries prediction. A review of the literature. Community Oral Dent Epidemiol 1998; 26: 361-71.

19. Vanobbergen J, Martens L, Lesaffre E, Bogaerts K, Declerck D. Assessing risk indicators for dental Caries in the primary Dentition. Community Oral Dent Epidemiol 2001; 29: 424-34.

20. Declerck D, Leroy R, Martens L, Lesaffre E, Garcia-Zattera MJ, Broucke V. Factors associated with prevalence and severity of caries experience in preschool children. Community Dent Oral Epidemiol 2008; 36: 68-78.

21. Öhlund I, Holgerson PL, Bäckman B, Lind T, Hernell O, Johansson I. Diet Intake and Caries Prevalence in Four-Year-Old Children Living in a Low-Prevalence Country. Caries Res 2006; 41(1): 26-33.

22. Karjalainen S, Söderling E, Sewón L, Lapinleimu H, Simell O. A Prospective study on sucrose consumption, visible plaque and caries in children from 3 to 6 years and of age. Community Dent Oral Epidemiol 2001; 29: 136-42.

23. Anderson M, Stecksen-Blicks, Stenlund H, Ranggard L, Tsilingaridis G, Mejare I. Detection of Approximal Caries 5 year - Old Swedish Children, Caries Res 2005; 39: 92-99.

24. Pienihakkinen K, Jokela J, Alanen P. Assessment of Caries risk in Preschool Children. Caries Res 2005; 38: 56-162.

25. Perinetti G Caputi S, Varvara G. Risk/Prevention indicators for the Prevalence of Dental Caries in Schoolchildren: Results from the Italian OHSAR Survey. Caries Res 2005; 39: 9-19.

26. Schwartz J. Sociodemographic and psychosocial factors in childhood as predictors of adult mortality. J. of Health 1995; 85 (9):1237-45.

27. Febres C, Echeverri E, Keene H. Parental awareness, habits, and social factors and their relationship to baby bottle tooth decay. Ped Dent 1997; 19 (1): 36.

28. Finlayson T, Siefert K, Ismail A, Sohn W. Psychosocial factors and early childhood caries among low - income African - American children in Detroit. Community Dent Oral Epidemiol 2007; 35: 439-448.

29. Marsh PD. Dental Plaque as a Microbial Biofilm. Caries Res 2004; 38: 204-211.

30. Nascimiento F, Mayer MPA, Pontes P, Pignatari ACC, Weckx LLM. Caries prevalence levels of Mutans Streptecocci, and gingival and plaque and indices in 3 to 5 years old mouth breathing children. Caries Res 2004; 38 (14): 572-575.

31. Dos santos M, Dos santos L, Francisco SB, Cury JA. Relationship among Dental Plaque composition Daily Sugar exposure and Caries in the Primary Dentition. Caries Res 2002; 36: 347352. 